\title{
Infection of the lymphatic system by Aureobasidium pullulans in a patient with erythema nodosum leprosum
}

\section{Authors}

Orlando Oliveira de Morais ${ }^{1}$

Cláudia Porto ${ }^{2}$

Anglya Samara Silva Leite Coutinho $^{1}$

Carmélia Matos Santiago Reis

Marcus de Melo Teixeira ${ }^{4}$ Ciro Martins Gomes

${ }^{1} \mathrm{MD}$; Dermatology Residency Program, University Hospital of Brasilia, Universidade de Brasília (UnB), Brasília, Brazil ${ }^{2} \mathrm{MD}, \mathrm{MSc}$; Department of Dermatology, University Hospital of Brasilia, UnB, Brasília, Brazil ${ }^{3} \mathrm{MD}, \mathrm{PhD}$; Laboratory of Mycology, University Hospital of Brasilia, UnB, Brasília, Brazil

${ }^{4} \mathrm{MSc}$., Laboratory of Molecular Biology, UnB, Brasília, Brazil
Submitted on: $12 / 08 / 2010$ Approved on: 02/14/2011

\section{Correspondence to:}

Cláudia Porto

Serviço de Dermatologia

Hospital Universitário de Brasília - UnB

SGAN 605

Avenida L2 Norte.

Asa Norte, Brasília

Distrito Federal, Brazil

70910-900

Phone/Fax:

+55 61 3448-5415

caca.porto@uol.com.br

We declare no conflict of interest.

\begin{abstract}
Aureobasidium pullulans is a causal agent of phaeohyphomycosis, occasionally found in men and animals. As an agent of different opportunistic fungal processes, it may cause fungemia, systemic infections and abscesses in different viscera. This paper aims to report a case of a patient with infection of the lymphatic system by A. pullulans. A 23-year-old patient being treated for erythema nodosum leprosum presented a 60-day complaint of daily fever, hoarseness, odynophagia and weight loss. Laboratory tests showed pancytopenia with severe neutropenia, cervical adenomegaly and solid contrast uptake lesion in the oropharyngeal region. Due to neutropenia and sepsis the patient was initially treated with cefepime and vancomycin, but there was no clinical improvement. Lymph node puncture-aspiration showed yeast-form fungus identified as A. pullulans by sequencing ITS region. The patient was treated with amphotericin B deoxycholate, leading to complete recovery of bone marrow function and regression of adenomegaly and the oropharyngeal lesion.
\end{abstract}

Keywords: HIV; pneumonia; macrolides; mortality.

[Braz J Infect Dis 2011;15(3):288-292] [OElsevier Editora Ltda.

\section{INTRODUCTION}

Aureobasidium pullulans is a fungal agent of universal distribution, an occasional causing agent of phaeohyphomycosis in human beings and animals. It is a black yeast-form fungus that causes many opportunistic fungal processes and owing to its capacity to produce extracellular biodegradable polysaccharide pullulan, as well as many different hydrolytic enzymes (e.g. amylase, proteinase, cellulase), it is considered an agent of great biotechnological importance. ${ }^{1}$

A. pullulans is an opportunistic agent rarely associated with infections in human beings and its pathogenic mechanisms are not very well known. However, infections located in the most varied organic topographies have already been associated with the agent, among which there are keratomycosis, spleen and mandible abscesses, meningitis, skin mycosis and peritonitis. ${ }^{2-6}$

Systemic fungal infections are relatively common in patients receiving broad spectrum antibiotics, using invasive devices, as well as those immunosuppressed. Among febrile neutropenic patients, systemic fungal infection is identified in about $10 \%$ of non-responders to initial antibacterial therapy, and in these patients opportunistic fungi such as Candida spp. and Asper- gilus spp. are the isolated agents in $80 \%$ to $90 \%$ of the cases. ${ }^{8}$ However, rare cases of septicemia by $A$. pullulans have also been reported. ${ }^{5,-12}$

The purpose of the present study was to report a case of a patient who had lymphatic system lesions which were attributed to infection by A. pullulans.

\section{CASE REPORT}

Male, 23-year-old patient, coming from Brasília, were being followed up by the Dermatology Division, University Hospital of Brasilia. The patient had received treatment for lepromatous leprosy seven years ago, although due to the erythema nodosum leprosum, he was still taking thalidomide $(200 \mathrm{mg} /$ day $)$ and prednisone ( $5 \mathrm{mg} /$ day) to achieve clinical control. For a period of 60 days the patient had been presenting symptoms of daily fever, hoarseness, odynophagia and weight loss which lead to the diagnosis of bacterial pharyngotonsillitis. The patient was initially treated as an outpatient having received prescription of amoxacilin, cephalexin and then clindamycin and ciprofloxacin, but with no symptoms relief.

At the hospital admission, the patient was markedly asthenic, febrile $\left(39^{\circ} \mathrm{C}\right)$ and had 
abnormality of vocal timbre. Upon clinical examination, he presented significant increase of volume and erythema of the palatine tonsils, as well as painful cervical adenomegaly. Clinical thoracic and abdominal examination were normal, except for dullness to percussion over Traube's space.

Laboratory tests showed a white blood cells count with pancytopenia and severe neutropenia, no findings in chest $\mathrm{x}$-ray and no liver or kidney abnormalities. Thereafter, the detection of febrile neutropenia, in a context of sepsis with probable oropharyngeal focus, led to immediate introduction of antibiotic therapy with cefepime and vancomycin ( $2 \mathrm{~g}$ IV BID and $1 \mathrm{~g}$ IV BID, respectively). As the patient's health status kept on deteriorating even after 48 hours of antibiotics use, and considering that the patient came from a kala-azar endemic region, amphotericin B deoxycholate (IV, $50 \mathrm{mg} /$ day) was associated to the treatment.

Further radiological investigation using CT scan showed a contrast-uptake solid lesion, involving the left side of the soft palate and palatoglossal and palatopharyngeal arches, measuring $30 \mathrm{~mm}$ in diameter, associated with multiple non-confluent adenomegaly of bilateral cervical chains (Figure 1). Abdominal CT scan confirmed the clinical findings, showing a mild increase in spleen volume. Myelogram showed hypocellular bone marrow with shift to the left, compatible with bone marrow aggression, without parasites or fungi in the analyzed sample. A rapid rK39 immunochromatographic dipstick test (Kala-azar Detect $^{\text {tw }}$ Rapid Test, InBios International, Seattle, WA, USA) was also performed and turned out negative. Blood cultures did not grow fungal or bacterial agents and laryngoscopy revealed no findings suggestive of neoplasm.

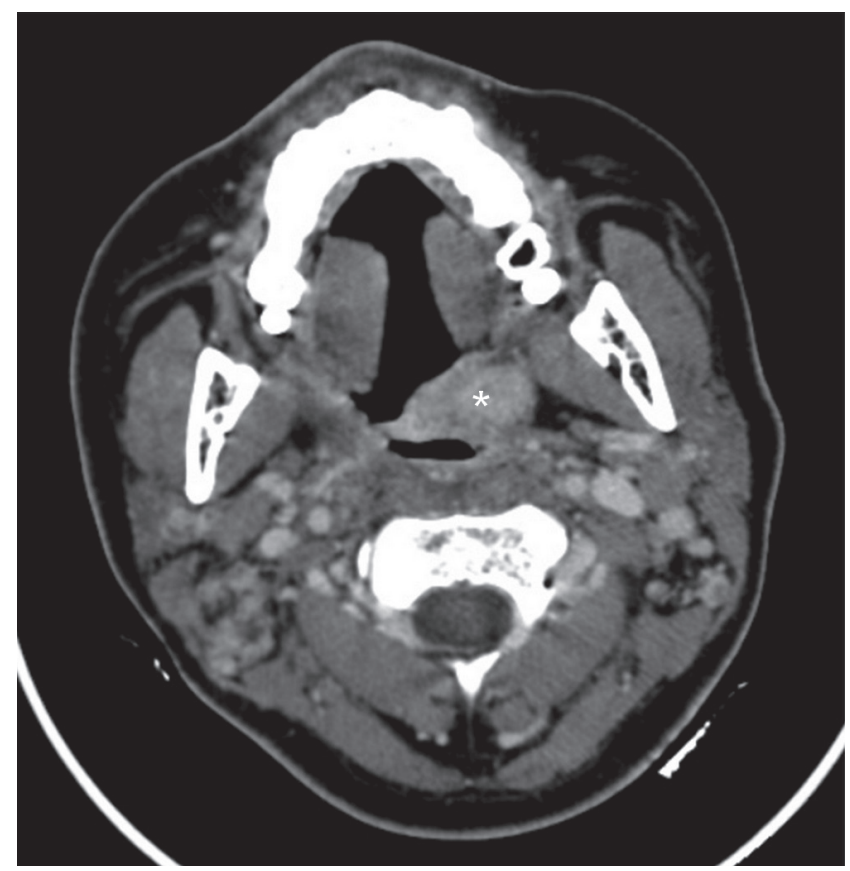

Figure 1: CT scan with contrast of the neck. High-enhanced exophytic lesion in the soft palate region on the left. Presence of multiple enlarged lymph nodes of the neck chains $\left(^{*}\right)$.
Viral serology (hepatitis B and C, HIV) was negative.

Therefore, cervical lymph node fine needle aspiration was undertaken and the analysis of the collected material showed presence of many fungal yeast-form structures with sprouting that were phagocytized by reticular cells (Figure 2). At that time, Paracoccidioidis brasiliensis was ruled out by PCR. The culture of the same material revealed growth of yeast-form fungal colony, comprised by hyaline septated hyphae given rise synchronically to multiple conidia. Some attempts were made to define the etiological agent using molecular markers. PCR amplification and sequencing of intergene region ITS1/2 of gene $18 \mathrm{~S}$ of the DNA extracted from the isolated colony led to the identification of $A$. pullulans as the putative pathogen. The fragment of sequenced ITS region (HUB_PAC1) presented 98\% identification with the environmental strain of $A$. pullulans SN22 (GENBANK access number: FJ515165), as well as with the clinical strain UWFP 769 (GENBANK access number: AY213639), showing a high level of preservation of sequenced nucleotides when they were aligned (Figure 3). After 30 days of incubation at $35^{\circ} \mathrm{C}$, the fungal colony started to produce a dark pigment (Figure 4) with presence of black septated hyphae, characteristic of A. pullulans.

The patient progressed with remarkable clinical recovery and had remission of fever and neutropenia after the $2^{\text {nd }}$ and $12^{\text {th }}$ day of amphotericin B use, respectively. After a total dose of $1,850 \mathrm{mg}$, administered in 40 days, the patient had already showed complete remission of adenomegaly and almost complete bone marrow recovery, persisting with mild anemia $(\mathrm{Hb}=11.8 \mathrm{~g} / \mathrm{dL})$. At that time, the patient was discharged using fluconazole at $300 \mathrm{mg}$, PO, twice a week for 8 weeks. Three months after the end of treatment, the patient was asymptomatic and had complete bone marrow recovery. Thalidomide was maintained during the whole period of treatment $(100 \mathrm{mg} /$ day $)$.

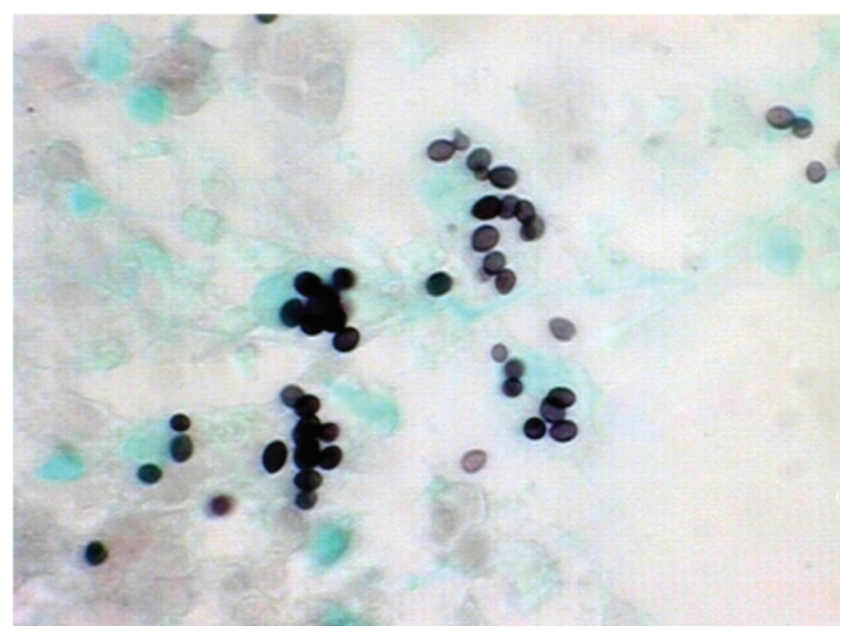

Figure 2: Cytology of lymph node aspirate. Many yeast-like fungal forms of round and oval shape and varied sizes are seen. Some fungal structures demonstrate polar sprouting (Grocott; 400X). 
HUB_PAC1

A. pullulans SN22

A. pullulans_UWFP769

HUB PAC1

A. pullulans SN22

A. pullulans UWFP769

HUB_PAC1

A. pullulans SN22

A. pullulans_UWFP769

HUB_PAC1

A. pullulans_SN22

A. pullalans_UWFP769

HUB_PAC1

A. pallalans SN22

A. pullulans_UWFP769

HUB_PAC1

A. pallulans SN22

A. pullulans UWFP769

$10 \quad 20$

30

40

50

60

70

80

90

100 $\ldots \ldots \ldots|\ldots| \ldots|\ldots| \ldots|\ldots| \ldots|\ldots| \ldots|\ldots| \ldots|\ldots| \ldots|\ldots| \ldots|\ldots| \ldots|\ldots| \ldots|\ldots| \ldots|\ldots| \ldots \mid$ GGGTGCTCAGCGCCCGACCTCCAACCCTTTGTTGTTAAAACTACCTTGTTGCTTTGGCGGGACC-GCTCGGTCTCGAGCCGCTGGGGATTCGTCCCAGGC GGGTGCTCAGCGCCCGACCTCCAACCCTTTGTTGTTAAAACTACCTTGTTGCTTTGGCGGGACC-GCTCGGTCTCGAGCCGCTGGGATTCGTCCCAGGC

110 120 130 140 150 160 170 180 190 200 $\ldots \ldots|\ldots| \ldots|\ldots| \ldots|\ldots| \ldots|\ldots| \ldots|\ldots| \ldots|\ldots| \ldots|\ldots| \ldots|\ldots| \ldots|\ldots| \ldots|\ldots| \ldots|\ldots| \ldots|\ldots| \ldots \mid$ GAGCGCOCTC GACCGCCCCCAGATC GAGCGCCGCCAGAGTTAAACCAAACTCTTGTTATTTAACCGGTCGTCTGAGTTAAAATTTTTAAATAAATCAAAACTTTCAACAACGGATCTCTTGGTTC

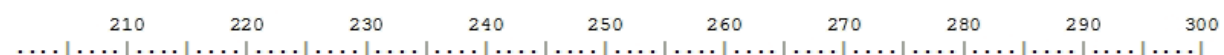
TCGCATCGATGAAGAACGCAGCGAATGCGATAAGTAATGTGAATTGCAGAATTCAGTGAATCATCGAATCTTTGAACGCACATTGCGCCCCTTGGTATT TCGCATCGATGAAGAACGCAGCGAAATGCGATAAGTAATGTGAATTGCAGAATTCAGTGAATCATCGAATCTTTGAACGCACATTGCGCCCCTTGGTATT TCGCATCGATGAAGAACGCAGCGAAATGCGATAAGTAATGTGAATTGCAGAATTCAGTGAATCATCGAATCTTTGAACGCACATTGCGCCCCTTGGTATT

$$
310
$$$$
320
$$$$
330
$$$$
340
$$

350

360

370

380

390

400 CCGAGGGGCATGCCTGTTCGAGCGTCATTACACCACTCAAGCTATGCTTGGTATTGGGTGCGTCCTTAGTTGGGCGCGCCTCAAAGACCTCGGCGAGGC CCGAGGGGCATGCCTGTTCGAGCGTCATTACACCACTCAAGCTATGCTTGGTATTGGGTGCCGTCCTTAGTTGGGCGCGCCTCAAAGACCTCGGCGAGGC CCGAGGGGCATGCCTGTTCGAGCGTCATTPCACCACTCAAGCTATGCTTGGTATTGGGTGCCGTCCTT AGTTGGGCGCGCCTTAAAGACCTCGGCGAGC

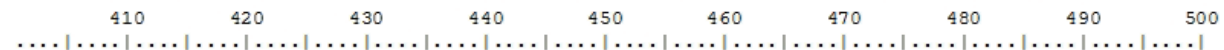
$\ldots \ldots|\ldots| \ldots|\ldots| \ldots|\ldots| \ldots|\ldots| \ldots|\ldots| \ldots|\ldots| \ldots|\ldots| \ldots|\ldots| \ldots|\ldots| \ldots|\ldots| \ldots|\ldots| \ldots|\ldots|$ CTCACCGGCTTAGGCGTAG ATTATTCGAACGTCTGTCAAAGGAGAGGACTTCTGCCGACTGAAACCTTTTATTTTTCTAGGTTGACCTCGGATC CTCACCGGCTTTAGGCGTAGTAGAATTTATTCGAACGTCTGTCAAAGGAGAGGACTTCTGCCGACTGAAACCTTTTATTTTTCTAGGTTGACCTCGGATC CTCACCGGCTTTAGGCGTAGTAGAATTTATTCGAACGTCTGTCAAAGGAGAGGACTTCTGCCGACTGAAACCTTT-ATTTTTCTAGGTTGACCTCGGATC

$$
510
$$

$\ldots|\ldots| \ldots|\ldots| \ldots$

AGGTAGGGATACCCGCTGAACT

AGGTAGGGATACCCGCTGAACT

AGGTAGGGATACCCGCTGAACT

Figure 3: Alignment of sequencing of regions ITS 1/2 of gene $18 \mathrm{~S}$ A. pullulans. Sequence HUB_PAC1, corresponds to studied strain, shows high level of similarity when compared to environmental strain SN22 (GENBANK access number: FJ515165) and strain of clinical origin UWFP769 (GENBANK access number: AY213639).

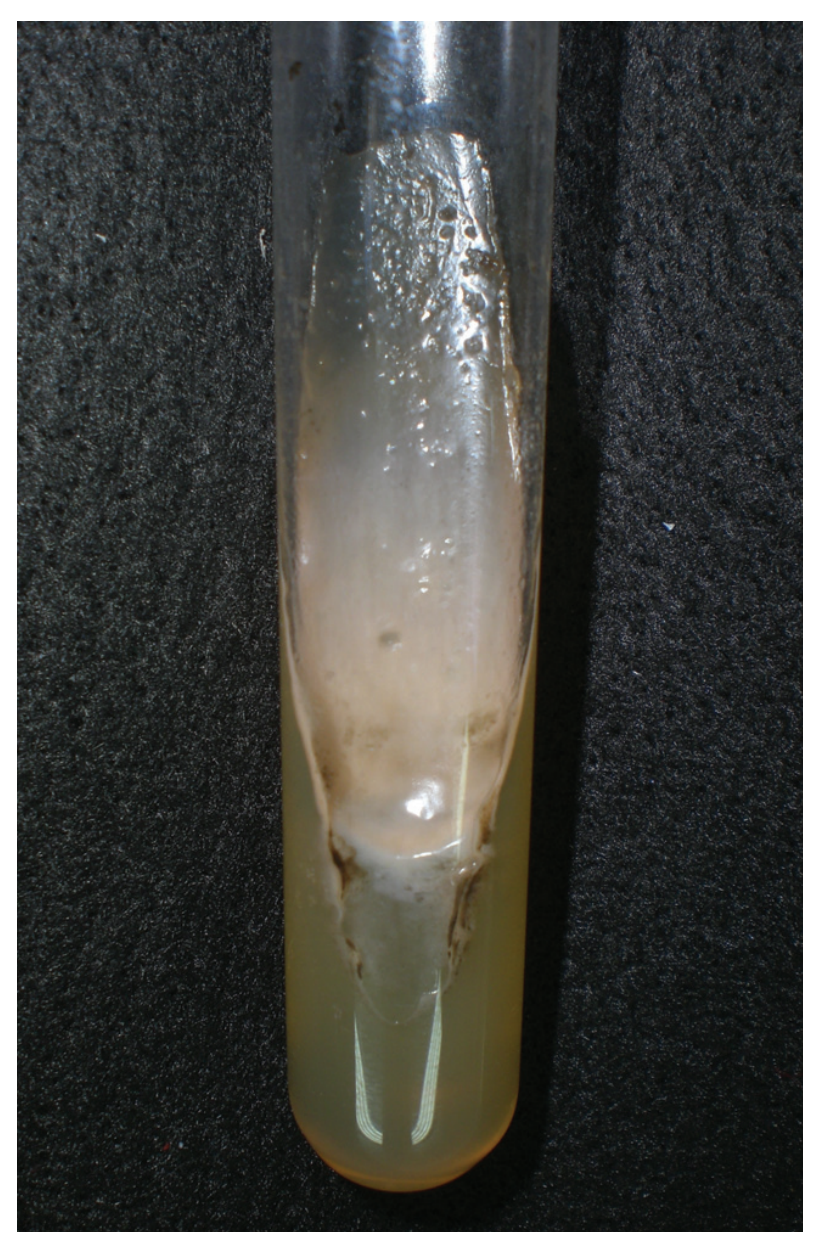

Figure 4: Macroscopy of fungal colony obtained in the material from lymph node aspirate. Yeast-like colonies of beige color, with initial points of dark pigment in areas after 30 days of incubation (agar-Sabouraud, $35^{\circ} \mathrm{C}$ ). 


\section{DISCUSSION}

A. pullulans is a saprophyte filamentous fungus of universal distribution, easily isolated in airborne samples, from superficial layers of the ground, domestic waste and leaves surface. It is a dematiaceous fungus, which presents colonies of quick growth, preferably at $25^{\circ} \mathrm{C}$, capable of producing black pigment similar to melanin, which is deposited on the cell wall and may act as virulence factor inhibiting phagocytosis by the host's immune system. ${ }^{13} \mathrm{~A}$. pullulans is normally considered a contaminant of biological samples, but the isolation from sterile sites should be valued given that the agent may act as an opportunistic agent.

When isolated in a culture, $A$. pullulans presents colonies initially yeast-form, yellowish, beige or light brown formed by dense groups of conidia synchronically originated from undifferentiated hyaline hyphae. As a result of maturation of colonies, hyphae accumulate pigment and become dark brown. ${ }^{7,11}$ The diagnosis is made by isolation and morphological characterization in specific culture medium which may be successfully studied, but their yeast-form shape may be mistaken by other yeasts of Dothideomycete class. ${ }^{2,6}$

The constant advance of medical science has provided increased survival to severely ill patients who in some situations require aggressive treatments, which commonly lead to iatrogenic immunosuppression, the use of invasive intrabody devices, and the need to use broad spectrum antibacterial agents for prolonged periods of time. Nevertheless, all those conditions are recognized as risk factor for fungal infections and have contributed for the increase in frequency of infections by opportunistic fungal agents. ${ }^{14}$ There are seven reported cases of systemic disseminated infections by A. pullulans as a result of isolation of the agent in blood culture. Joshi et al. ${ }^{5}$ reported a case of a 11-year-old patient who had Fanconi anemia and, during the conditioning regimen for the performance of bone marrow transplantation, because of bone marrow suppression, developed a febrile episode followed by cutaneous lesions caused by A. pullulans, which was later isolated in blood culture collected from the venous central catheter. Another case was reported by Kaczmarski et al., ${ }^{12}$ who described a 28 -year-old patient with acute myeloid leukemia, who had neutropenia secondary to chemotherapy and reported isolation of $A$. pullulans in a blood sample collected from Hickman catheter used to infuse chemotherapy agents. The involvement of these neutropenic patients, as well as the large proportion of cases of peritonitis caused by dialysis catheter infection, confirm the role of invasive intrabody devices and immunosuppression states as facilitators of these systemic infections. ${ }^{15}$

In the initial assessment, the significant findings of patient were increased volume and erythema of palatine tonsils, cervical adenomegaly and splenomegaly, associated with hematological manifestation of pancytopenia. The severe and extensive involvement of the lymphohematopoietic system, together with absence of clinical improvement after 48 hours after onset of antimicrobials, prompted to the introduction of amphotericin $\mathrm{B}$, in addition to broad the possibilities of differential diagnoses.

Clinical data supported by the patient coming from regions endemic of both kala-azar and South-American paracoccidioidomycosis and also the suspicion of hematological neoplasm (lymphoma) or squamous cell carcinoma, led to immediate myelogram and lymph node puncture-aspiration for diagnostic investigation.

Even though the myelogram had shown neither fungal nor parasite agents or neoplastic findings, mycological diagnosis was suggested through fine needle aspiration of a cervical lymph node, which showed yeast-like fungal structures with sprouting which were phagocytized by host macrophages. From a cytopathology standpoint, owing to absence of demaceous hyphae in the material, it was not possible to define an etiological classification due to the similarity of $A$. pullulans conidia and those of other yeast-like fungi. ${ }^{16}$ In the past, the production of pigment was a key factor for the identification of demaceous species such as Aureobasidium from culture medium, but it is known that this event occurs only as a result of hyphae aging, which delays or even causes diagnostic errors. In view of these factors, the use of gene sequencing in regions ITS1/2 showed quick and precise etiological diagnosis of $A$. pullulans, which reinforces the efficacy of this method as a diagnostic tool for fungal species, in agreement with the data reported in the literature. ${ }^{17,18}$

Salkin et al. ${ }^{2}$ reported a case of fungal invasion of the lymphatic system, causing the formation of spleen abscess in a patient systematically affected by histiocytic lymphoma. The entry of the infection was not identified by the authors, but the profound immunesuppression caused by the lymphoma seemed to have played a predisposing role in the secondary infection by A. pullulans. In another case, disseminated infection of the fungus was reported in a 28-year-old patient who had multiple exposed fractures of the left femur and tibia because of a car accident. ${ }^{11}$ The exposure of the wounds to the ground during the car accident was reported by the authors as a likely source of infection, since the ground is a known reservoir of the agent. A retrospective analysis of our case made us believe that the lesions in the oral mucosa could have served as an entry point for the infection in our patient, since there were no other specific foci. Moreover, immune studies have shown that patients with polar forms of lepromatous leprosy and erythema nodosum leprosum present a pattern of immune response mediated by Th2 lymphocytes with the production of IL4 and IL10 cytokines. Such cytokines induce the humoral immune response via CD8(+) lymphocytes and also seem to lead to negative regulation of Toll-like $2 / 1$ receptors, determining an ineffective response against Hansen bacilli. ${ }^{19}$ Both the history of lepromatous leprosy of the patient and the concurrent use of 
thalidomide at the moment of infection, a drug known for its anti-TNF-alpha action, made us advocate that the patient might have presented a predominantly humoral immune response pattern to A. pullulans. These factors may have facilitated the dissemination of infection in view of low levels of Th1-type (INF gamma, TNF-alpha) proinflammatory cytokines, widely known for their important immune response against fungal agents. ${ }^{20}$

Treatment of A. pullulans infections, either localized or disseminated, is not a consensus since up to the present day treatment recommendations are based on case reports and case series. In the present case, the total dose of $1,850 \mathrm{mg}$ of conventional amphotericin B was enough for complete remission of cervical adenomegaly and bone marrow recovery. Nevertheless, the patient's prompt recovery after introduction of the antifungal, even before the isolation of the agent, reinforces the importance of following up evidence-based protocols for sepsis management, especially in the immunosuppressed or neutropenic hosts.

Therefore, even in the absence of fungal structures visualized in the analysis of bone marrow aspirate, we believe that the manifestation of pancytopenia presented by the patient may have been secondary to bone marrow invasion by the agent. To support this hypothesis, we excluded other infectious agents that may have determined similar clinical and laboratory manifestations. Alternatively, medications such as clindamycin, ${ }^{21}$ ciprofloxacin, ${ }^{22}$ and thalidomide ${ }^{23}$ had been associated with episodes of bone marrow suppression, but considering the rarity of these reports and the complete hematological recovery of the patient, even during maintenance of thalidomide, we think it is unlikely that the manifestation of marrow suppression had been an adverse reaction to the use of these drugs.

The difficulty to define the diagnosis of A. pullulans infection, as reported by us and other contemporary authors, ${ }^{15,16}$ owing to agent polymorphism or the large range of differential diagnoses, has confirmed the great value of molecular biology as a diagnostic tool.

\section{ACKNOWLEDGEMENTS}

We would like to thank Martha Mariana de Almeida Santos Arruda, MD, for her collaboration with the investigation and for the scientific support in managing the hematology abnormalities.

\section{REFERENCES}

1. Zalar P, Gostincar C, de Hoog GS et al. Redefinition of $\mathrm{Au}$ reobasidium pullulans and its varieties. Stud Mycol. 2008; 61(1):21-38.

2. Salkin IF, Martinez JA, Kemna ME. Opportunistic infection of the spleen caused by Aureobasidium pullulans. J Clin Microbiol. 1986; 23(5):828-31.
3. Koppang HS, Olsen I, Stuge U, Sandven P. Aureobasidium infection of the jaw. J Oral Pathol Med. 1991; 20(4):191-5.

4. Arranz Sánchez DM, de la Calle M, Martín-Díaz MA et al. Subcutaneous mycosis produced by Aureobasidium pullulans in a renal transplant recipient. J Eur Acad Dermatol Venereol. 2006; 20(2):229-30.

5. Joshi A, Singh R, Shah MS, Umesh S, Khattry N. Subcutaneous mycosis and fungemia by Aureobasidium pullulans: a rare pathogenic fungus in a post allogeneic BM transplant patient. Bone Marrow Transplantat. 2010; 45:203-4.

6. Mise N, Ono Y, Kurita N et al. Aureobasidium pullulans peritonitis: case report and review of the literature. Perit Dial Int. 2008; 28(6):679-81.

7. Lacaz CS, Porto E, Martins JEC, Heins-Vaccari EM, Melo NT. Tratado de Micologia Médica. São Paulo: Sarvier, 2002.

8. Figuera Esparza M, Carballo M, Silva M, Figueredo A, Avilán J. Microbiological isolates in patients with febrile neutropenia and hematological neoplasias. Rev Esp Quimioter. 2006; 19(3):247-51.

9. Hawkes M, Rennie R, Sand C, Vaudry W. Aureobasidium pullulans infection: fungemia in an infant and a review of human cases. Diagn Microbiol Infect Dis. 2005; 51(3):209-13.

10. Girardi LS, Malowitz R, Tortora GT, Spitzer ED. Aureobasidium pullulans septicemia. Clin Infect Dis. 1993;16:338-9.

11. Bolignano G, Criseo G. Disseminated nosocomial fungal infection by Aureobasidium pullulans var. melanigenum: a case report. J Clin Microbiol. 2003; 41:4483-5.

12. Kaczmarski EB, Liu Yin L, Tooth JA, Love EM, Delamore IW. Systemic infection with Aureobasidium pullulans in a leukaemic patient. J Infect. 1986; 13:289-91.

13. Langfelder K, Streibel M, Jahn B, Haase G, Brakhage AA. Biosynthesis of fungal melanins and their importance for human pathogenic fungi. Fungal Genet Biol. 2003; 38:143-58.

14. Del Palacio A, Villar J, Alhambra A. Epidemiology of invasive candidiasis in pediatric and adult populations. Rev Iberoam Micol. 2009; 26(1):2-7.

15. Huang YT, Liaw SJ, Liao CH et al. Catheter-related septicemia due to Aureobasidium pullulans. Int J Infect Dis. 2008; 12(6):e137-9.

16. Hofman V, Butori C, Long E et al. Aureobasidium pullulans contamination in bronchial aspirates mimicking cryptococcosis: a rare diagnostic pitfall. Pathology 2008; 40(7):729-32.

17. Rakeman JL, Bui U, Lafe $\mathrm{K}$ et al. Multilocus DNA sequence comparisons rapidly identify pathogenic molds. J Clin Microbiol. 2005; 43(7):3324-33.

18. Iwen PC, Hinrichs SH, Rupp ME. Utilization of the internal transcribed spacer regions as molecular targets to detect and identify human fungal pathogens. Med Mycol. 2002; 40:87109.

19. Modlin RL. The innate immune response in leprosy. Curr Opin Immunol. 2010; 22(1):48-54.

20. MacCallum DM. Massive induction of innate immune response to Candida albicans in the kidney in a murine intravenous challenge model. FEMS Yeast Res. 2009; 9(7):1111-12.

21. Bubalo JS, Blasdel CS, Bearden DT. Neutropenia after singledose clindamycin for dental prophylaxis. Pharmacotherapy 2003; 23(1):101-3.

22. Pons M, del Blanco J, Mateo L, Juanola X. Ciprofloxacininduced neutropenia: an infrequent adverse effect. Med Clin (Barc) 1991; 96(8):318-9.

23. Andersohn F, Konzen C, Garbe E. Systematic review: agranulocytosis induced by nonchemotherapy drugs. Ann Intern Med. 2007; 146(9):657-65. 